\title{
Lipophilic Ligand Efficiency as a Useful Metric in Hit and Lead Optimization
}

\author{
Hamid Irannejad* \\ Department of Medicinal chemistry, Faculty of Pharmacy, Mazandaran University of Medical Sciences, Sari, Iran
}

*Corresponding author: Hamid Irannejad, Department of Medicinal chemistry, Faculty of Pharmacy, Mazandaran University of Medical Sciences, Sari, Iran, Tel: 0098 9124572673; E-mail: irannejadhamid@gmail.com

Received: 17 Jan, 2019 | Accepted: 31 Jan, 2019 | Published: 06 Feb, 2019

Citation: Irannejad H (2019) Lipophilic Ligand Efficiency as a Useful Metric in Hit and Lead Optimization. J Med Chem Drug Des 2(1): dx.doi.org/10.16966/2578-9589.112

Copyright: (c) 2019 Irannejad H. This is an open-access article distributed under the terms of the Creative Commons Attribution License, which permits unrestricted use, distribution, and reproduction in any medium, provided the original author and source are credited.

\begin{abstract}
Traditional medicinal chemistry has directed hits and leads toward larger size and more lipophilic compounds during optimization. Avoiding molecular obesity has been a major challenge in drug discovery. Nowadays, useful metrics are developing to substantially help medicinal chemists to find more efficient ligands of own targets. In this essay, ligand efficiency metrics are introduced and discussed which are used to measure the extent of potency gain of a ligand originating from increasing of its lipophilicity.
\end{abstract}

Fragment based drug discovery (FBDD) has overcome major problems of lead discovery in high throughput screening (HTS) due to its higher chance of hit finding. The higher hit rate of FBDD is attributed to its smaller chemical space and higher chance of complementarity of fragments to the shape of binding site [1].

In a FBDD project, as hits are grown and linked together to become leads and drug candidates, their lipophilicity and molecular size increases inevitably to increase affinity and potency. In this sense, increase in lipophilicity has many drawbacks such as off-target and non-selective binding, side effects and violations of Lipinski's rule of 5 or drug likeness properties. Similarly, increasing in molecular weight lowers solubility and permeability of oral drugs and reduces druglikeness properties [2].

Normally, most medicinal chemists only consider potency and try to increase it during hit and lead optimization or when studying structure-activity relationship. Notably, lipophilicity should be considered along with potency variations to avoid lipophilicity violations. Accordingly, scientists have developed ligand efficiency (LE) and lipophilic ligand efficiency (LLE) concepts as a useful metric in lead discovery and optimization and these parameters can be calculated by the following equations.

$$
\begin{aligned}
& \mathrm{LE}=-\Delta \mathrm{G}_{\mathrm{b}} / \mathrm{HA} \\
& \mathrm{LLE}=-\log \text { (potency) }-\log \mathrm{D}
\end{aligned}
$$

In order to prevent hit compounds to become larger during optimization, ligand efficiency (LE) can be used to assess binding affinity contribution of each non-hydrogen atom (heavy atom). This parameter was first proposed by Kuntz and coworkers [3] and was further developed by Hann and Keseru [4] and then Hopkins et al. [5]. This metric parameter is calculated by negative of the free energy of binding $\left(-\Delta \mathrm{G}_{\mathrm{b}}\right)$ divided by the number of heavy atoms. The accepted value of LE during lead optimization should be maintained above 0.3 . It is recommended to start with a fragment or hit which has a higher value of LE because LE normally decreases as long as optimization continues. Undoubtedly, lipophilic ligand efficiency (LLE) is the most important metric in hit optimization and is calculated by subtracting $\log \mathrm{D}$ value of a compound from the negative logarithm of its potency (usually in $\mathrm{IC}_{50}$ or $\mathrm{K}_{\mathrm{i}}$ form in molar concentration) against a specific target. This concept was first proposed by Leeson and Springthorpe [6]. With this metric tool, we can say whether increase in potency is mediated by an increase in lipophilicity or not. Compounds which have an increase in potency during optimization but with constant LLE, shows that possibly this increase in potency is the result of increasing in lipophilicity. Specifically, increasing in LLE indicates that increasing in potency is much more than the increase in lipophilicity $(\log D)$. Several studies have reported the correlation of compound high lipophilicity to the likelihood of off-target binding or promiscuous binding to proteins. This phenomenon usually tends to adverse effects, toxicity, reduced selectivity, and poor ADME profile [7,8].

Someone may think that increasing polarity of a molecule would be a reasonable way to increase LLE but in fact, decreasing compound lipophilicity often results in potency loss due to desolvation penalty or loss of hydrophobic binding site interactions. Therefore, adding a polar group in order to reduce lipophilicity $(\log \mathrm{D})$ should be done with care and is recommended to occur at a point where locates in solvent-accessible region of a molecule. Polar groups at solventexposed region of a molecule do not need to be desolvated when bound to the target because it is outside of binding site space. Therefore, a common strategy to increase LLE without changing potency or affinity is modification of solvent accessible part of a molecule by reducing $\log \mathrm{D}$ [9]. The suggested range of LLE is 5-7 
or even higher and to achieve this range, $\log \mathrm{D}$ must be smaller than 3. Many publications and investigations have recommended $\log \mathrm{D}$ of lead and drug candidates in the range of 1-3 for optimum ADME and pharmacokinetic properties. Correspondingly, high LLE values favor specific binding of compounds to the respected target more efficiently. Finally, making decision only based on potency value is not recommended at all for hit or lead optimization process due to molecular obesity problems and simultaneous use of ligand efficiency metrics are advised throughout the project.

\section{References}

1. Orita M, Warizaya M, Amano Y, Ohno K, Niimi T (2009) Advances in fragment-based drug discovery platforms. Expert Opin Drug Discov 4: $1125-1144$

2. Keseru GM, Makara GM (2009) The influence of lead discovery strategies on the properties of drug candidates. Nat Rev Drug Discov 8: 203-212.

3. Kuntz ID, Chen K, Sharp KA, Kollman PA (1999) The maximal affinity of ligands. Proc Natl Acad Sci U S A 96: 9997-10002.

4. Hann MM, Keseru GM (2012) Finding the sweet spot: the role of nature and nurture in medicinal chemistry. Nat Rev Drug Discov 11: 355-365.
5. Hopkins AL, Keseru GM, Leeson PD, Rees DC, Reynolds CH (2014) The role of ligand efficiency metrics in drug discovery. Nat Rev Drug Discov 13: 105-121.

6. Leeson PD, Springthorpe B (2007) The influence of drug-like concepts on decision-making in medicinal chemistry. Nat Rev Drug Discov 6: 881-890.

7. Kramer JA, Sagartz JE, Morris DL (2007) The application of discovery toxicology and pathology towards the design of safer pharmaceutical lead candidates. Nat Rev Drug Discov 6: 636-649.

8. Meanwell NA (2016) Improving Drug Design: An Update on Recent Applications of Efficiency Metrics, Strategies for Replacing Problematic Elements, and Compounds in Nontraditional Drug Space. Chem Res Toxicol 29: 564-616.

9. Johnson TW, Tanis SP, Butler SL, Dalvie D, Delisle DM, et al. (2011) Design and synthesis of novel N-hydroxy-dihydronaphthyridinones as potent and orally bioavailable HIV-1 integrase inhibitors. J Med Chem 54: 3393-3417. 